\title{
Listeria monocytogenes Internalin and E-cadherin: From Bench to Bedside
}

\author{
Matteo Bonazzi ${ }^{1,2,3}$, Marc Lecuit ${ }^{4,5,6}$, and Pascale Cossart ${ }^{1,2,3}$ \\ ${ }^{1}$ Institut Pasteur, Unité des Interactions Bactéries-Cellules, Paris, F-75015 France \\ ${ }^{2}$ Inserm, U604, Paris, F-75015 France \\ ${ }^{3}$ INRA, USC2020, Paris, F-75015 France \\ ${ }^{4}$ Institut Pasteur, Groupe Microorganismes et Barrières de I'Hôte, Paris, F-75015 France \\ ${ }^{5}$ Inserm Avenir U604, Paris, F-75015 France \\ ${ }^{6}$ Université Paris Descartes, Centre d'Infectiologie Necker-Pasteur, Service des Maladies Infectieuses et \\ Tropicales, Hôpital Necker-Enfants malades, Assistance Publique-Hôpitaux de Paris, Paris, F-75015 France \\ Correspondence: pcossart@pasteur.fr, mlecuit@pasteur.fr
}

Listeria monocytogenes is a Gram-positive bacterium responsible for a severe infection associated with different clinical features (gastroenteritis, meningoencephalitis, and abortion in pregnant women). These pathologies are caused by the unusual capacity of the bacterium to cross three host barriers during infection and to invade nonphagocytic cells. To invade host cells, Listeria uses two proteins, InIA and InIB, which have specific receptors on the host-cell surface, E-cadherin and Met, respectively. Here, we discuss the specificity of the $\operatorname{InI} \mathrm{A}-\mathrm{E}$-cadherin interaction, the signaling cascade activated on E-cadherin engagement by $\operatorname{InIA}$, and the role of $\operatorname{InIA}$ and E-cadherin in the breaching of host barriers and the dissemination of the infection.

isteriosis is a potentially lethal food-borne Linfection with a mortality rate up to $30 \%$. It has emerged as a significant human infection in industrialized countries along with the development of large-scale agro-industrial plants and refrigerated food. Opposite to most food-borne infections, listeriosis is rare but potentially very severe, because it remains often under-diagnosed at its early stages (Lecuit 2007). The etiological agent of listeriosis is Listeria monocytogenes, a Gram-positive bacterium that contaminates meat, dairy products, and ready to eat food. Upon ingestion of contaminated food, L. monocytogenes can colonize the intestine and gives rise to gastroenteritis in case of the absorption of a high inoculum. Strikingly, L. monocytogenes has the capacity to cross the intestinal barrier and disseminate to the mesenteric lymph nodes, spleen, and liver. In immunocompromised individuals, L. monocytogenes may replicate in the spleen and liver, cause prolonged and sustained bacteremia, cross the blood-brain barrier and the placental barrier, and disseminate to the brain and

Editors: W. James Nelson and Elaine Fuchs

Additional Perspectives on Cell Junctions available at www.cshperspectives.org

Copyright (C) 2009 Cold Spring Harbor Laboratory Press; all rights reserved; doi: 10.1101/cshperspect.a003087

Cite this article as Cold Spring Harb Perspect Biol 2009;1:a003087 
placenta, causing meningitis, encephalitis, abortion in pregnant women, and neonatal infections (Hamon et al. 2006; Bonazzi et al. 2009).

The capacity of $L$. monocytogenes to cross multiple host barriers relies on the ability of the bacterium to invade nonphagocytic cells, such as epithelial cells, by interacting with host cell-surface receptors. Adhesion to host cells is a key step underlying bacterial pathogenicity and it is required to counteract the mechanical clearance at tissue surfaces provided by intestinal peristaltism and blood flow. Internalization allows persistence in a shielded niche, away from the soluble effectors of the host immune system (Cossart and Sansonetti 2004; PizarroCerda and Cossart 2006), and access to target organs, as illustrated by $L$. monocytogenes (Bonazzi et al. 2009).

Bacterial surface proteins that engage host receptors are generally called adhesins, although it is now clear that many of them not only mediate adhesion, but also bacterial internalization (Boyle and Finlay 2003; Hauck et al. 2006; Pizarro-Cerda and Cossart 2006). Adhesion to and internalization of L. monocytogenes within epithelial cells is mainly mediated by two bacterial surface protein members of the internalin family, namely internalin (InlA) and InlB, that use E-cadherin and Met as receptors, respectively, on the surface of host cells (Hamon et al. 2006; Pizarro-Cerda and Cossart 2006; Bonazzi et al. 2009). Upon receptormediated internalization, L. monocytogenes is engulfed into the cell and becomes surrounded by a tight phagocytic vacuole that the bacterium can lyse by means of the pore-forming toxin listeriolysin O (LLO). Once free in the cytoplasm of the host cell, L. monocytogenes uses the protein ActA to harness the actin polymerization machinery and facilitate its intracellular movement via the formation of so-called actin "comet tails." Actin-based motility is fundamental for L. monocytogenes direct cell-to-cell spread, a typical feature that allows the dissemination of the infection to neighboring cells via the formation of plasma membrane protrusions. Once internalized by neighboring cells, L. monocytogenes is confined in a double-membrane vacuole from which it escapes to restart its life cycle (Fig. 1) (Hamon et al. 2006).

Its remarkable adaptation to the cellular environment and its capacity to exploit cellular receptor-mediated signaling pathways and the actin polymerization machinery have made L. monocytogenes an exceptional tool for the study of a wide array of cellular functions (Cossart and Sansonetti 2004; Bonazzi and Cossart 2006; Hamon et al. 2006; PizarroCerda and Cossart 2006; Veiga and Cossart 2005a; Bonazzi et al. 2009). Here, we review the interaction of InlA with E-cadherin, the signaling pathway initiated by this interaction that results in the internalization of L. monocytogenes, and the role of InlA-E-cadherin interaction during listeriosis.

\section{THE L. MONOCYTOGENES SURFACE PROTEIN InIA}

As introduced above, internalization of $L$. monocytogenes within nonphagocytic cells is mediated by the two bacterial surface proteins InlA and InlB. In vitro, these bacterial proteins are sufficient to mediate adhesion and internalization into host cells, as bacteria expressing either one of these proteins as well as beads coated with either InlA or InlB are efficiently internalized in nonphagocytic cells as long as their respective receptor is expressed (Lecuit et al. 1997; Pizarro-Cerda et al. 2002).

The targets of InlA and InlB on the surface of the host cell are the adhesion molecule E-cadherin and the hepatocyte growth factor (HGF) receptor Met, respectively (Hamon et al. 2006). Met is ubiquitously expressed, allowing InlB to mediate bacterial internalization in a large number of cell types, whereas InlA shows a more stringent cell tropism, as E-cadherin is only expressed by a limited number of cells of epithelial origin.

InlA and InlB are part of a large family of proteins, the internalin family, composed of 25 proteins sharing a common architecture that includes a signal peptide at the amino-terminal and a number of 22 amino acid leucine-rich repeats (LRR) that can vary from three in the case of Lmo2445 to 28 in the case of InlI. 
Listeria monocytogenes Internalin and E-cadherin: From Bench to Bedside

LRR repeats are common to a large number of proteins and are involved in protein-protein interactions (Kobe and Deisenhofer 1994). Downstream of the LRR, internalins display several other regions that are less conserved within the family and can confer specific functions to different members of the family (Bierne et al. 2007). InlA is an 800 amino-acid protein containing 15 LRRs. Downstream of the LRR region, InlA has an inter-repeat (IR) region that has been shown fundamental for the binding of the LRR repeats domain to E-cadherin (Lecuit et al. 1997). An LPXTG motif for the anchoring of InlA at the bacterial cell wall is present at the carboxy-terminal followed by a sorting peptide (Hamon et al. 2006).
The first evidence that indicated that InlA might act as a L. monocytogenes virulence factor derived from studies on the L. monocytogenes strain LO28, in which it has been observed that it harbors a frameshift mutation in the inlA gene that results in a nonsense codon at position 1729. This generates a truncated open reading frame encoding a protein of $63 \mathrm{kDa}$ that lacks the LPXTG motif (Jonquieres et al. 1998). As a consequence, in the LO28 strain, InlA can not be detected on the surface of the bacteria but is found released in the supernatant (Jonquieres et al. 1998), and LO28 invasion of epithelial cell lines is far lower than other strains expressing a functional InlA. Epidemiological studies performed on 300
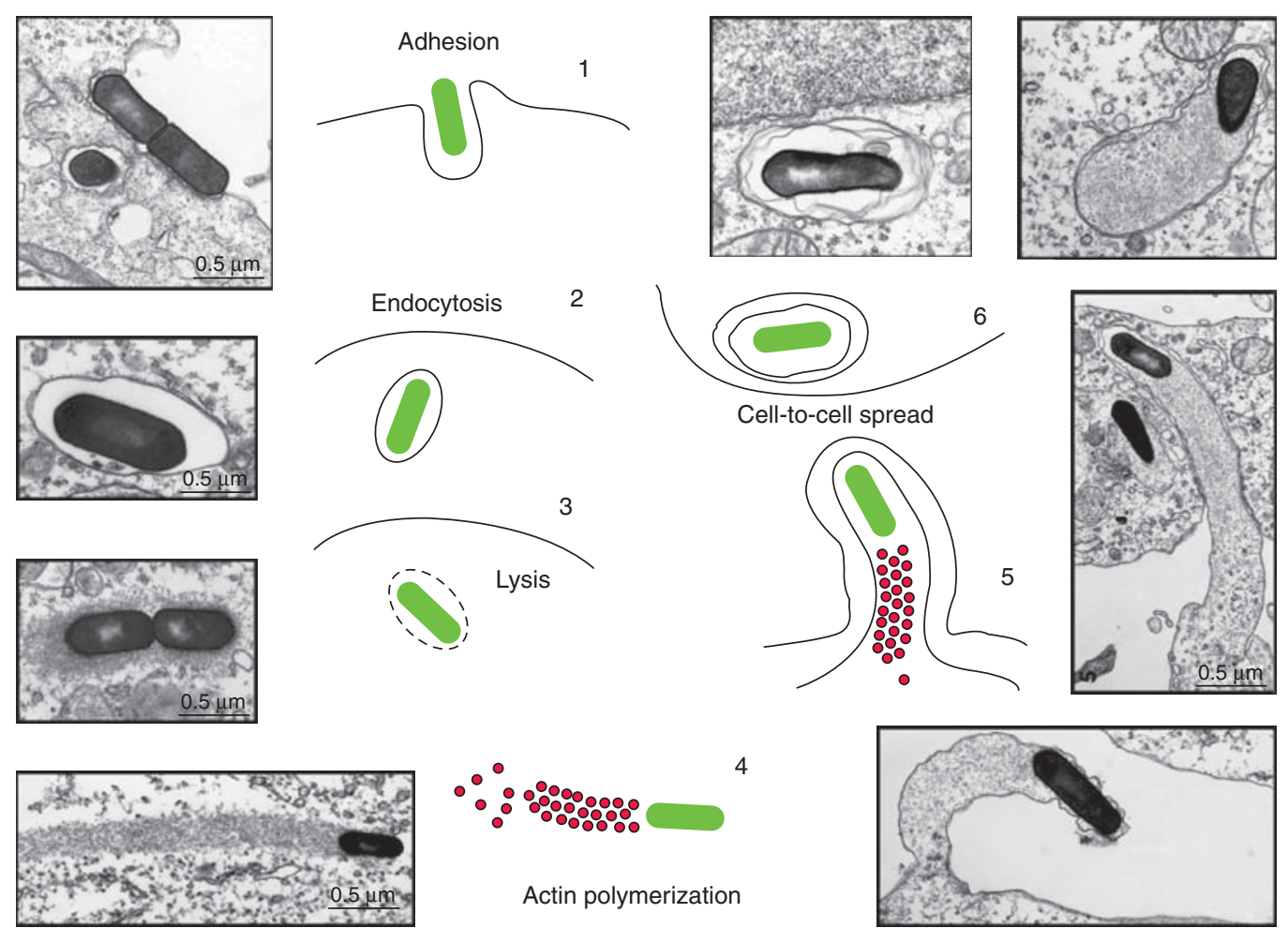

Actin polymerization

4
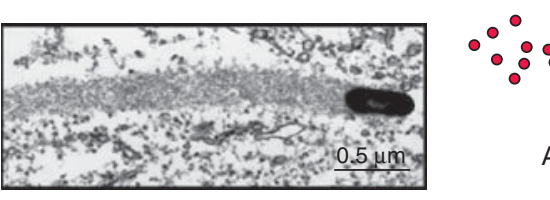

Figure 1. The cell cycle of L. monocytogenes. (1) L. monocytogenes adheres to the surface of epithelial cells via the interactions of the surface proteins InlA and InlB with E-cadherin and the Met receptor, respectively. (2) On internalization, L. monocytogenes is engulfed in a phagocytic vacuole. (3) L. monocytogenes lyses vacuolar membranes by means of the toxin LLO. (4) L. monocytogenes uses the protein ActA to harness the actin polymerization machinery and facilitate its intracellular movement via the formation of so-called actin "comet tails." (5) L. monocytogenes exploits actin-based motility for direct cell-to-cell spread to allow the dissemination of the infection to neighboring cells via the formation of plasma membrane protrusions. (6) Once internalized by neighboring cells, L. monocytogenes is confined in a double-membrane vacuole from which it escapes to restart its life cycle. 
clinical strains obtained in France and 150 strains from food isolates showed that, at least in food isolates, the truncation in the InlA gene is not a rare event (Jonquieres et al. 1998). More recently, a thorough epidemiological study showed that full length internalin was identified in $96 \%$ of the clinical strains and in $65 \%$ of the strains derived from food isolates (Jacquet et al. 2004). These observations indicated that InlA might serve as a virulence determinant of L. monocytogenes in humans, a hypothesis that has now been confirmed in humanized animal models (see the following). The crystal structure of InlA, alone or in complex with the EC1 domain of E-cadherin, has been solved (Schubert et al. 2002). The LRR domain of InlA consists of fifteen and a half 22-residue repeats that form a right-handed curved solenoid structure. Each repeat begins with a $\beta$ strand of five residues and these strands combine to form a 16 -stranded $\beta$ sheet that is twisted out of a central plane to form a pseudo-helical surface. The cavity thus created forms the binding site for the EC1 domain of E-cadherin (Fig. 2B).

\section{E-CADHERIN, THE RECEPTOR FOR INLA, MEDIATES L. MONOCYTOGENES ENTRY INTO HOST CELLS}

E-cadherin belongs to the family of classical cadherins that mediate the formation of adherens junctions, $\mathrm{Ca}^{2+}$-dependent intercellular adhesion sites that develop between polarized epithelial cells (Troyanovsky 2005; Meng and Takeichi 2009). Cadherins have been classified into several subfamilies on the basis of shared properties and sequence similarities. Type I cadherins, also called "classical" cadherins, all possess five 110-amino-acid immunoglobulinlike extracellular domains (EC1 to EC5) with a conserved tryptophan in position 2 and interact with actin through their intracellular domain (Shapiro and Weis 2009). This subfamily includes E-cadherin, which is found in epithelial cells, $\mathrm{N}$-cadherin that was first detected in neuronal cells, C-cadherin expressed in the Xenopus embryo, and P-cadherin on the placenta and other cell types. T-cadherins are still classified as members of the type I subfamily, although they lack the transmembrane and cytoplasmic domain and are tethered to the plasma membrane via a GPI-anchor (Ranscht and Dours-Zimmermann 1991; Patel et al. 2003). Type II cadherins differ from members of the type I subfamily in that they have two conserved tryptophan residues in position 2 and 4 . They also interact with the actin cytoskeleton via specific adaptors and the most common member of this subfamily is VEcadherin, which is expressed by endothelial cells (Vestweber et al. 2009; Delva et al. 2009). Desmosomal cadherins form another subfamily of proteins: they share with type I cadherins the presence of $5 \mathrm{EC}$ repeats and a conserved tryptophan in position 2. Nevertheless, members of this family differ in the cytoplasmic region that allows the interaction with intermediate filaments instead of actin. Desmoglein and Desmocollin belong to this family and as their names indicate are localized at the level of desmosomes (Green and Gaudry 2000). The Protocadherins subfamily can be further divided in $\alpha, \beta$, and $\gamma$ protocadherins. They all present six EC repeats and they are expressed at the level of synaptic complexes in neurons (Kohmura et al. 1998; Patel et al. 2003).

E-cadherin is a protein of 882 amino acids with a 555-amino-acids-long amino-terminal extracellular domain, a transmembrane domain, and a relatively short cytoplasmic domain of 152 amino acids. The extracellular portion is comprised of five 110-amino-acid repeats (EC1 to EC5), immunoglobulin-like domains involved in intercellular homotypic interactions and in intracellular clustering (Troyanovsky 2005). The analysis of their crystal structure revealed that multiple cadherin domains form $\mathrm{Ca}^{2+}$-dependent rodlike structures with a $\mathrm{Ca}^{2+}$-binding pocket at the interface between two contacting EC domains (Shapiro and Weis 2009). Besides E-cadherin itself, the only known eukaryotic E-cadherin ectodomain interactor identified is $\alpha E \beta 7$ integrin (Karecla et al. 1996), which is solely expressed in epithelial lymphocytes, and has E-cadherin as its unique binding partner. E-cadherin is also the receptor for Listeria 
Listeria monocytogenes Internalin and E-cadherin: From Bench to Bedside

A

InIG Lmo0262

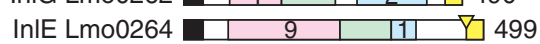

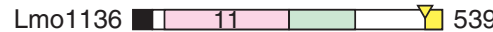

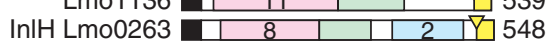

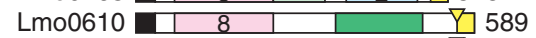

Lmo1289 \begin{tabular}{|l|l|l|l|l}
8 & 7 & & $Y$ \\
\hline
\end{tabular} 593

Lmo1290 \begin{tabular}{l|l|l|l|l}
\hline & 8 & & & $Y$ \\
\hline
\end{tabular} 598

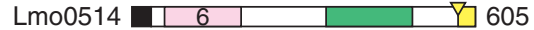

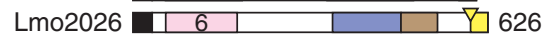

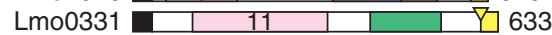

Lmo0732 \begin{tabular}{l|l|l|l|l|l}
\hline & 8 & & & & $Y$
\end{tabular} 638

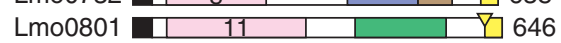

InIA Lmo0433 \begin{tabular}{l|l|l|l}
\hline 15 & & 3 & Y \\
\hline
\end{tabular}

InIF Lmo0409 \begin{tabular}{l|l|l|l|l|l}
\hline 13 & & 4 & $Y$ \\
\hline
\end{tabular}

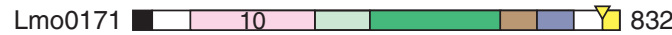

InlJ Lmo2821 \begin{tabular}{l|l|l|l|l} 
& & & & \\
\hline
\end{tabular}

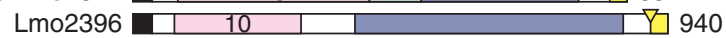

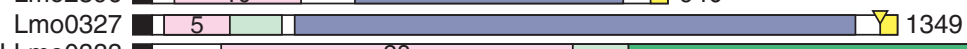

Inll Lmo0333

InIB Lmo0434

Lmo0549 \begin{tabular}{c|c|c|c|c|c|} 
GW'GW GW \\
WxL WxL
\end{tabular}

InIC Lmo1786 $\square=6 \quad \square 296$

Lmo2445 $\square 300$

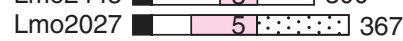

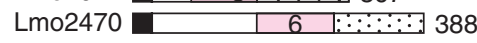

B

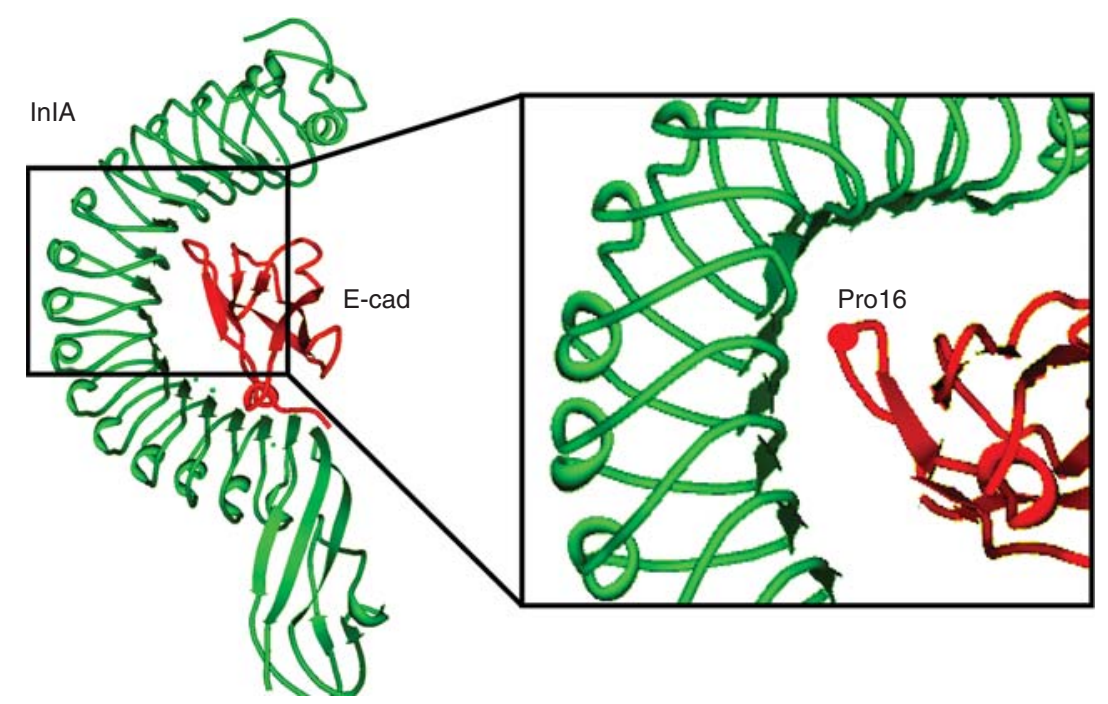

Figure 2. The internalin family and InlA. (A) Schematic representation of the internalin family of proteins of L. monocytogenes. Homologous regions are color coded as indicated in the legend. Numbers within different domains indicate the number of repeats (Bierne et al. 2007). (B) Crystal structure of InlA in complex with the EC1 domain of E-cadherin. The LRR domain of InlA consists of fifteen and a half 22-residue repeats that form a right-handed curved solenoid structure. Each repeat begins with a $\beta$ strand of five residues: these strands combine in a 16-stranded $\beta$ sheet that forms a pseudo-helical surface. The presence of a proline in position 16 of E-cadherin allows the terminal loop of E-cadherin to be hydrophobic and uncharged, therefore strengthening the interaction with InlA. 
InlA (Mengaud et al. 1996), and the Candida albicans invasin Als3 (Phan et al. 2007) and it is the target for the Bacterioides fragilis metalloprotease toxin BFT (Wu et al. 1998).

The cytoplasmic domain of E-cadherin is divided into a juxtamembrane domain that spans from aa 734 to aa 770 , approximately and a distal domain from aa 770 to 882 . Phosphorylation of the cytoplasmic domain regulates the binding of E-cadherin to its interactors (Fig. 3) (McCrea et al. 2009). The nonreceptor tyrosine kinase Src phosphorylates E-cadherin juxtamembrane domain at residues 753 and 754. This post-translational modification triggers the release of the E-cadherin direct interactor p120 catenin and the recruitment of the ubiquitin-ligase Hakai (Fujita et al. 2002; Reynolds 2007). In its E-cadherin bound state, p120 stabilizes E-cadherin at the plasma membrane by sterically impeding the binding of Hakai to E-cadherin. In its cytosolic form, p120 regulates cell motility by activating Rac1 and Cdc42 and inhibiting RhoA (Reynolds 2007). Upon E-cadherin phosphorylation, Hakai binds to and ubiquitinates E-cadherin, triggering its clathrin-mediated internalization (Fujita et al. 2002). Deletion of the last 35 amino acids of the cytoplasmic tail of E-cadherin impairs the binding to $\beta$-catenin (Ozawa et al. 1990; Lecuit et al. 2000) that binds $\alpha$-catenin, a key regulator of actin dynamics (Fig. 3).

Binding of E-cadherin to $\beta$-catenin plays a fundamental role in the regulation of adherens junction dynamics and is negatively regulated by phosphorylation of both E-cadherin and $\beta$-catenin. Casein-kinase-1-mediated serine phosphorylation of E-cadherin at residue 846 has been reported to impede binding to $\beta$-catenin (Dupre-Crochet et al. 2007). Constitutively phosphorylated E-cadherin at serine 846 fails to localize at adherens junctions and to bind $\beta$-catenin efficiently (DupreCrochet et al. 2007). Similarly, $\beta$-catenin can be phosphorylated by Src at tyrosine 654 (Roura et al. 1999) and by Met at tyrosine 142 (Brembeck et al. 2004), which induces the dissociation of $\beta$-catenin from both E-cadherin and $\alpha$-catenin. Phosphorylation of $\beta$-catenin at tyrosine 489 has been reported in neuronal cells, it is mediated by the tyrosine kinase Abl

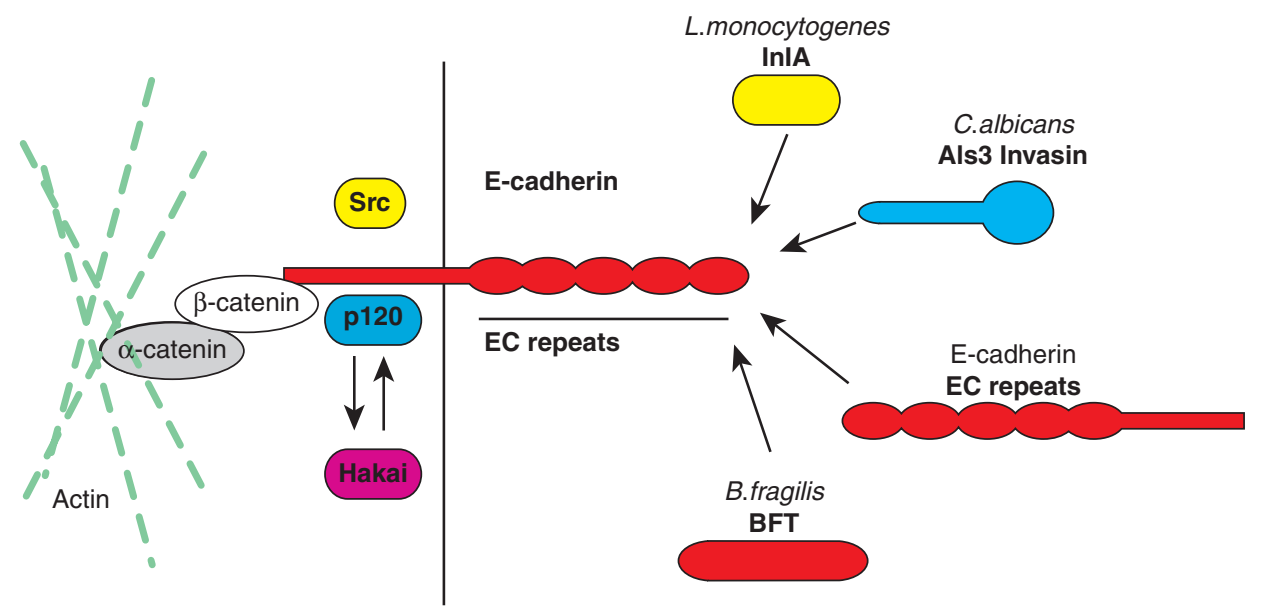

Figure 3. E-cadherin interactors. E-cadherin is a single-pass transmembrane protein with a 152-amino-acid intracellular domain and a 555-amino-acid extracellular domain. The intracellular domain of E-cadherin interacts with proteins of the catenin family. p120 catenin binds to the juxtamembrane domain of E-cadherin and stabilizes E-cadherin at the plasma membrane. On E-cadherin phosphorylation by the tyrosine kinase Src p120 is released and the ubiquitin-ligase Hakai can bind and ubiquitinate E-cadherin. $\beta$-catenin binds to the carboxy-terminal domain of E-cadherin and mediates the interaction with the actin cytoskeleton via $\alpha$-catenin. The extracellular domain of E-cadherin interacts with the EC domains of E-cadherin molecules from neighboring cells, it serves as the receptor for the L. monocytogenes surface protein InlA and the C. albicans invasin Als3, and is the target of the B. fragilis metalloprotease toxin BFT. 
on activation of the surface receptor Robo, and, it induces the dissociation of $\beta$-catenin from E-cadherin (Rhee et al. 2007). Most cellular $\beta$-catenin is associated with E-cadherin at adherens junctions. Cytoplasmic pools of $\beta$-catenin can be either phosphorylated at tyrosine 412 or nonphosphorylated and are rapidly translocated to the nucleus. Here, Wnt signaling can induce a conformational change in nonphosphorylated $\beta$-catenin, whereby its carboxyl terminus folds into a close conformation that impedes binding to E-cadherin and favors the binding to the T-cell factor (TCF) in a transcription complex (Gottardi and Gumbiner 2004; see Heuberger and Birchmeier 2009). Alternatively, in its phosphorylated form, $\beta$-catenin regulates transcription by binding BCL9-2, a homolog of the human oncogene product BCL9 (Brembeck et al. 2004).

A direct interaction between $\beta$-catenin and Met has also been documented and the regulation of such interaction depends on tyrosine residues 654 and 670 on $\beta$-catenin (Zeng et al. 2006). Of note, InlB, the other major L. monocytogenes internalin, is a potent activator of Met, suggesting a possible link between the two signaling pathways exploited by the bacterium for its internalization. Concerning the interactions of $\beta$-catenin with the actin cytoskeleton, it has been reported that $\alpha$-catenin switches from a monomeric form with a high affinity for $\beta$-catenin to a dimeric form that has a higher affinity for actin: This equilibrium would regulate actin dynamics at adherens junctions (Drees et al. 2005; Yamada et al. 2005). It has been recently shown that in Drosophila embryos, where homophilic E-cadherin interactions generate the so-called spot adherens junctions (SAJ), $\alpha$-catenin-mediated interaction of E-cadherin with actin is not required at the SAJ itself but rather at its sides to maintain the mosaic distribution of homo-E-cadherin clusters (Cavey et al. 2008).

\section{DOWNSTREAM EFFECTORS OF InIA-E-CADHERIN INTERACTION}

The observation that $\alpha$ - and $\beta$-catenin, as well as actin and p120 catenin, are efficiently recruited at the site of InlA-mediated bacterial entry (Lecuit et al. 2000), suggested not only that E-cadherin serves as a receptor for InlA, but also that on ligand/receptor engagement, L. monocytogenes is able to exploit the machinery involved in the formation of adherens junctions. A link between E-cadherin and the actin cytoskeleton is also fundamental: InlAmediated infection is impaired in cells expressing an E-cadherin mutant that lacks either the complete intracellular domain or the $\beta$ catenin-binding domain (Lecuit et al. 2000), which is in agreement with the previous observation that inhibiting actin polymerization by cytochalasin D impairs bacterial internalization (Gaillard et al. 1987). Interestingly, the deletion of the juxtamembrane domain of E-cadherin, important for p120 catenin binding, and E-cadherin endocytosis (Fujita et al. 2002), has no effect on the internalization of L. monocytogenes (Lecuit et al. 2000), which suggests the presence of an alternative pathway exploited by the bacterium. Indeed, our recent data indicate that in the case of the InlA pathway of L. monocytogenes, caveolin also participates in bacteria internalization and this pathway is independent of the presence of the E-cadherin juxtamembrane domain (Bonazzi et al. 2008).

Myosins were previously identified as important players in the stabilization of Ecadherin-mediated cell-cell junction and it has been proposed that their activity as actinbased motor proteins was necessary to generate the force that maintains mature adherens junctions (Vasioukhin and Fuchs 2001). Nonconventional myosin VIIa is also involved in InlA-mediated internalization of L. monocytogenes (Sousa et al. 2004). Myosin VIIa and the ubiquitous transmembrane protein vezatin were found to be recruited at the bacterial entry site when L. monocytogenes follows the InlA-mediated entry pathway and the inhibition of either protein severely affects bacterial internalization by the InlA pathway (Sousa et al. 2004). A relevant role of $\alpha$-catenin in the recruitment of vezatin and myosin VIIa was shown, stressing the relevance of catenins during InlA-mediated infections, not only as regulators of actin dynamics but also as 
scaffolding proteins for the recruitment of other components of the adherens junction machinery (Sousa et al. 2004).

The study of L. monocytogenes interactions with eukaryotic cells has also proven to be a powerful tool to identify new proteins involved in both bacterial internalization and adherens junction dynamics. The Rho GAP protein ARHGAP10 is a novel component of the adherens junction complex identified in a yeast twohybrid screen as an interactor of $\alpha$-catenin (Sousa et al. 2005). In cells that do not express E-cadherin, ARHGAP10 is predominantly localized at the Golgi complex, but in epithelial cells expressing E-cadherin, a large fraction of the protein colocalizes with E-cadherin, actin, and $\alpha$-catenin at adherens junctions (Sousa et al. 2005). In these cells, the knockdown of ARHGAP10 results in loss of $\alpha$-catenin localization at the adherens junctions, whereas E-cadherin localization is unaffected (Sousa et al. 2005). It has been proposed that ARHGAP10 recruits $\alpha$-catenin to newly formed cell-cell contacts once "passive" transcellular E-cadherin interaction has occurred, to mediate the maturation of adherens junctions (Sousa et al. 2005). In agreement with this hypothesis, ARHGAP10 colocalizes with $\alpha$-catenin and actin at the bacterial entry site during InlA-mediated infections. Furthermore, the inhibition of ARHGAP10 impairs the InlA-mediated internalization of L. monocytogenes (Sousa et al. 2005). ARHGAP10 has been also identified as the binding partner of GTP-bound Arf1 (and Arf6) at the Golgi complex where it plays an important role as a Cdc42-specific GAP protein that regulates actinmediated Golgi complex architecture (Dubois et al. 2005). Of note, when ARHGAP10 was identified as the binding partner for GTPbound Arf6, vezatin was also found among the screened preys (Dubois et al. 2005).

\section{LISTERIA TAKES CONTROL OF THE PHAGOCYTIC AND ENDOCYTIC MACHINERIES}

As for other bacteria, actin rearrangements are critical for internalization. In the case of
L. monocytogenes, the signaling that directly orchestrates actin remodeling and bacterial engulfment into host cells involves the Arp $2 / 3$ complex, a highly conserved actin nucleator, which is recruited at the bacterial entry site. Arp2/3 recruitment precedes actin cup formation around bacteria, in agreement with the idea that its function is required for actin polymerization (Sousa et al. 2007). Indeed, Arp2/3 inhibition impairs bacterial and InlA-coated bead internalization. A search for putative activators of Arp2/3 suggested that cortactin could be a candidate for the activation of Arp2/3, whereas WAVE and NWASP are not involved (Sousa et al. 2007).

Cortactin modulation of the actin cytoskeleton is regulated by the combined activity of Erk and Src kinases. It has been proposed that an initial phosphorylation by Erk at serines 405 and 418 liberates the SH3 domain of cortactin, otherwise folded in a close conformation on the proline-rich domain of cortactin (Campbell et al. 1999). In this conformation, cortactin is able to bind NWASP. Further phosphorylation of Src at tyrosines 421,466 , and 482 (Huang et al. 1998) impairs cortactin interaction with NWASP (Martinez-Quiles et al. 2004) and dramatically reduces the ability of cortactin to crosslink F-actin (Huang et al. 1997). In its Src-phosphorylated state, cortactin promotes cell migration (Huang et al. 1998). Of note, InlA-mediated L. monocytogenes internalization induces the activation of Src early during infection and the pharmacological inhibition of the Src family of tyrosine kinases inhibits entry as well as cortactin recruitment at the bacterial entry site (Sousa et al. 2007). Besides its possible role in the activation of cortactin, Src has been implicated in the initial phosphorylation of E-cadherin that initiates the internalization of the receptor from adherens junctions (Fujita et al. 2002). Src-mediated phosphorylation of E-cadherin is also triggered by InlA during bacterial infections (Bonazzi et al. 2008). This post-translational modification results in the recruitment of the ubiquitin-ligase Hakai at bacterial entry site and in the ubiquitination of E-cadherin, which is necessary for efficient bacterial internalization (Bonazzi et al. 2008). 
As mentioned above, Src-mediated phosphorylation of E-cadherin also induces the release of p120 catenin in the cytoplasm, where it activates Racl and Cdc42 (Reynolds 2007). The activation of Racl has been shown to have a key role in the recruitment of cortactin at the plasma membrane (Weed et al. 1998).

Upon InlB interaction with the Met receptor, L. monocytogenes can hijack the host ubiquitination machinery to enter epithelial cells via clathrin-dependent endocytosis (Veiga and Cossart 2005b). More recently, this observation has been extended, revealing that clathrindependent endocytosis is an entry pathway shared by a number of zippering bacteria (Veiga et al. 2007). This observation showed that the maximal size of particles that can be internalized via clathrin can exceed $100 \mathrm{~nm}$ and, in the case of InlA-mediated internalization, suggested a possible role of the endocytosis machinery in the establishment of adherens junctions (Veiga et al. 2007; Bonazzi et al. 2008). As recently shown, caveolin is required for E-cadherin clustering at the bacterial entry site (Bonazzi et al. 2008). Caveolin and clathrin may then mediate bacterial internalization (Fig. 4). Interestingly, both endocytic proteins are substrates of Src (Wilde et al. 1999; Joshi et al. 2008). Clathrin-mediated bacterial internalization specifically depends on the presence of the juxtamembrane domain of E-cadherin (Bonazzi et al. 2008). Clathrin and caveolin are also required for the internalization of InlA-coated beads and E-cadherin-coated beads (Bonazzi et al. 2008), suggesting that the endocytic machinery is involved in the initial steps of adherens junctions formation.

\section{FROM STRUCTURE TO PATHOGENESIS}

InlA interacts in a specific manner with EC1, the amino-terminal EC repeat of E-cadherin also involved in the initial interaction between E-cadherin molecules on the surface of adjacent cells (Lecuit et al. 1999). The region of the EC1 domain involved in InlA binding differs from that involved in the formation of homotypic interactions between E-cadherins, suggesting that E-cadherin molecules engaged in adherens junctions are still able to bind InlA. Despite the large degree of similarities between E-cadherin orthologs, InlA shows a species specificity for some E-cadherins, as its interaction is completely impaired in rat and mouse, whereas it is functional in other mammalian species such as humans, dogs, rabbits, guinea pigs, and gerbils (Lecuit et al. 1999). This species specificity depends on the nature of a single amino acid, a proline in position 16 within the $\mathrm{EC} 1$ domain of E-cadherin in permissive species, and a glutamic acid in nonpermissive mouse and rat E-cadherin (Lecuit et al. 1999). The E-cadherin loop that harbors a proline in position 16 is hydrophobic and uncharged, whereas a glutamic acid at this location results in a charged and hydrophilic region, suggesting that InlA/ E-cadherin interaction involves hydrophobic interactions. Of note, in humans, a proline in position 16 is present in all members of the classical cadherins. However, a conserved proline in position 16 , although necessary, is not the only critical residue for InlA interaction with the EC1 domain of cadherins, as InlA does not bind $\mathrm{N}$-cadherin, although it harbors a proline in position 16 (Mengaud et al. 1996).

These observations have been confirmed when the crystal structure of InlA, alone or in complex with the EC1 domain of E-cadherin, was solved (Fig. 2) (see previous discussion) (Schubert et al. 2002). Replacing proline 16 with glutamic acid in silico in the EC1 domain of E-cadherin, changes the hydrophilic properties of the binding site for InlA, therefore hampering a close interaction between receptor and ligand (Schubert et al. 2002). Overall, the affinity of InlA for E-cadherin is low and bacterial attachment and internalization is thought to reflect the cooperation of multiple interactions. It has been proposed that the lack of a high affinity binding may be of advantage for the bacterium, as it allows the potential dissociation of InlA from E-cadherin once the bacterium has been internalized, but this has not been investigated experimentally (Wollert et al. 2007a).

A key result was obtained when a transgenic mouse expressing human E-cadherin at the enterocyte level was created. Both the 
M. Bonazzi, M. Lecuit, and P. Cossart
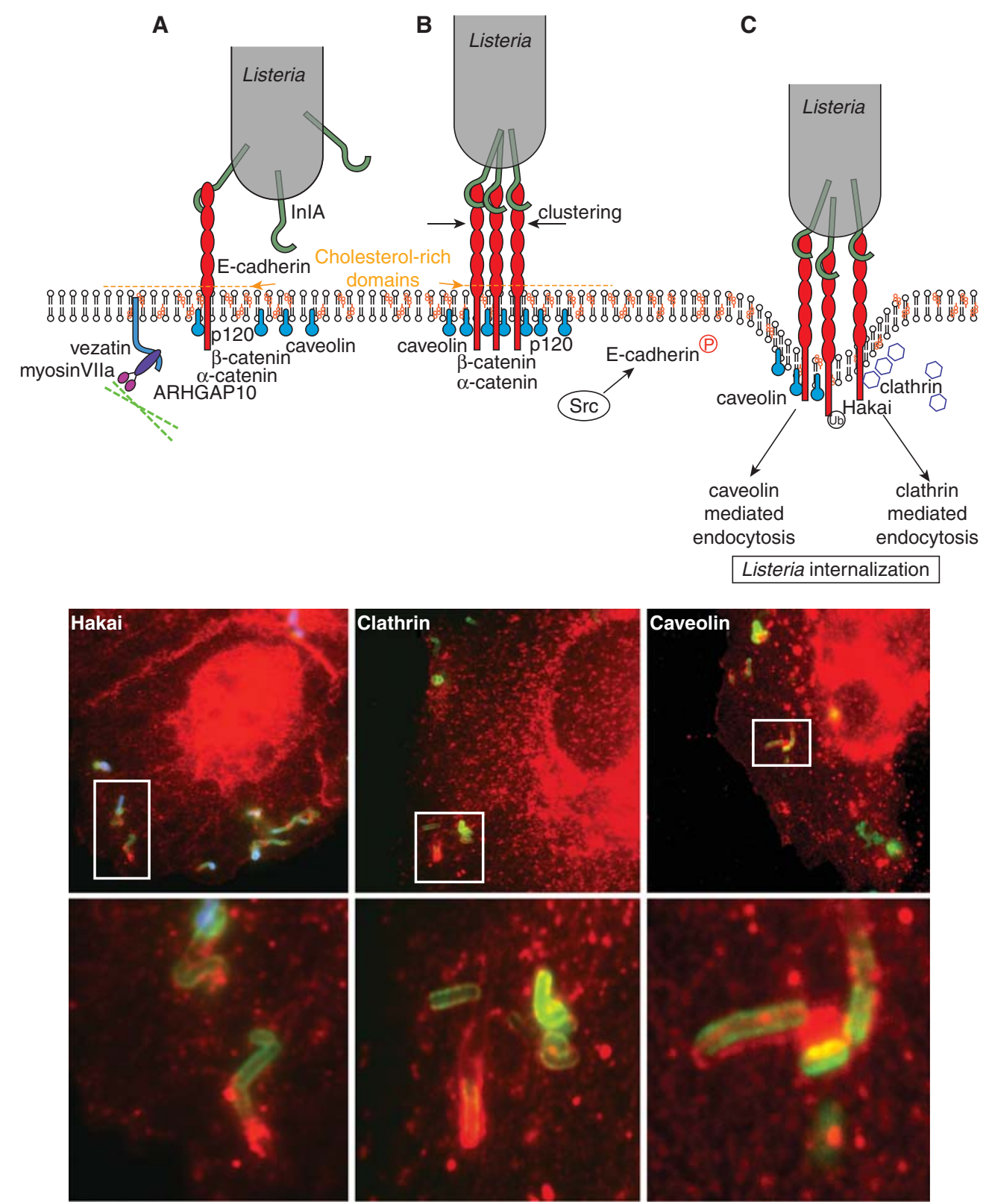

Figure 4. Dynamics of the InlA/E-cadherin interaction (upper panel). (A) On engagement of E-cadherin by InlA, the adherens junction machinery is activated, inducing the recruitment of the junctional proteins $\alpha$-catenin, p120 catenin, ARHGAP10, and myosin VIIa. (B) InlA interaction with E-cadherin induces the caveolin-dependent clustering of E-cadherin and the activation of the tyrosine kinase Src. $(C)$ The Src-dependent phosphorylation of E-cadherin triggers the recruitment of the ubiquitin-ligase Hakai and the ubiquitination of E-cadherin. E-cadherin ubiquitination induces the sorting of E-cadherin within clathrin-coated pits for bacterial internalization. Alternatively, E-cadherin may persist within caveolin-rich domains and bacterial internalization can occur via caveosomes. Bottom panels: Listeria (green) recruits Hakai (left panel), clathrin (middle panel), and caveolin (right panel) at the bacterial entry site when invading epithelial cells. Lower images are a magnified view of the highlighted areas. 
observation that proline 16 is conserved in E-cadherin of guinea pigs and the generation of these transgenic mice, allowed for the first time the study of InlA-mediated Listeria infections in a small animal model (Lecuit et al. 2001). These studies showed that InlA (Lecuit et al. 1997; Lecuit et al. 2001), but not InlB (Khelef et al. 2006), plays a critical role in the crossing of the intestinal barrier (Fig. 5). In contrast, when animals are infected intravenously, InlA plays no role, suggesting that InlA is a key virulence factor during the early stage of the infections (Lecuit et al. 2001).

More recently, InlA has been engineered so as to enhance its affinity for human Ecadherin, and this modified InlA also showed an ability to interact with mouse E-cadherin (Wollert et al. 2007b). In particular, replacing serine in position 192 with an asparagine bridges the gap between InlA and E-cadherin and the substitution of the tyrosine in position 369 with a serine allows the formation of a hydrogen bond. Of note, these mutations increase by four orders of magnitude the affinity of this "murinized" InlA ( $\left.\operatorname{InlA}^{\mathrm{m}}\right)$ for murine E-cadherin, which is in the same range of affinity than that of InlA with human E-cadherin (Wollert et al. 2007b). Under these conditions mice appear to become susceptible to InlA ${ }^{\mathrm{m}}$-mediated infection. Studies on the role of InlA ${ }^{\mathrm{m}}$ confirmed the previously described role of InlA during early oral infections (Lecuit et al. 2001; Wollert et al. 2007b). Surprisingly, whereas murinized InlA has a greatly increased binding affinity for hEcad, this does not translate into a significantly higher level of internalization in in vitro cultured cells expressing hEcad, such as Caco-2 cells (Wollert et al. 2007b).

Interestingly, although epidemiological data strongly argued for a role for InlA in
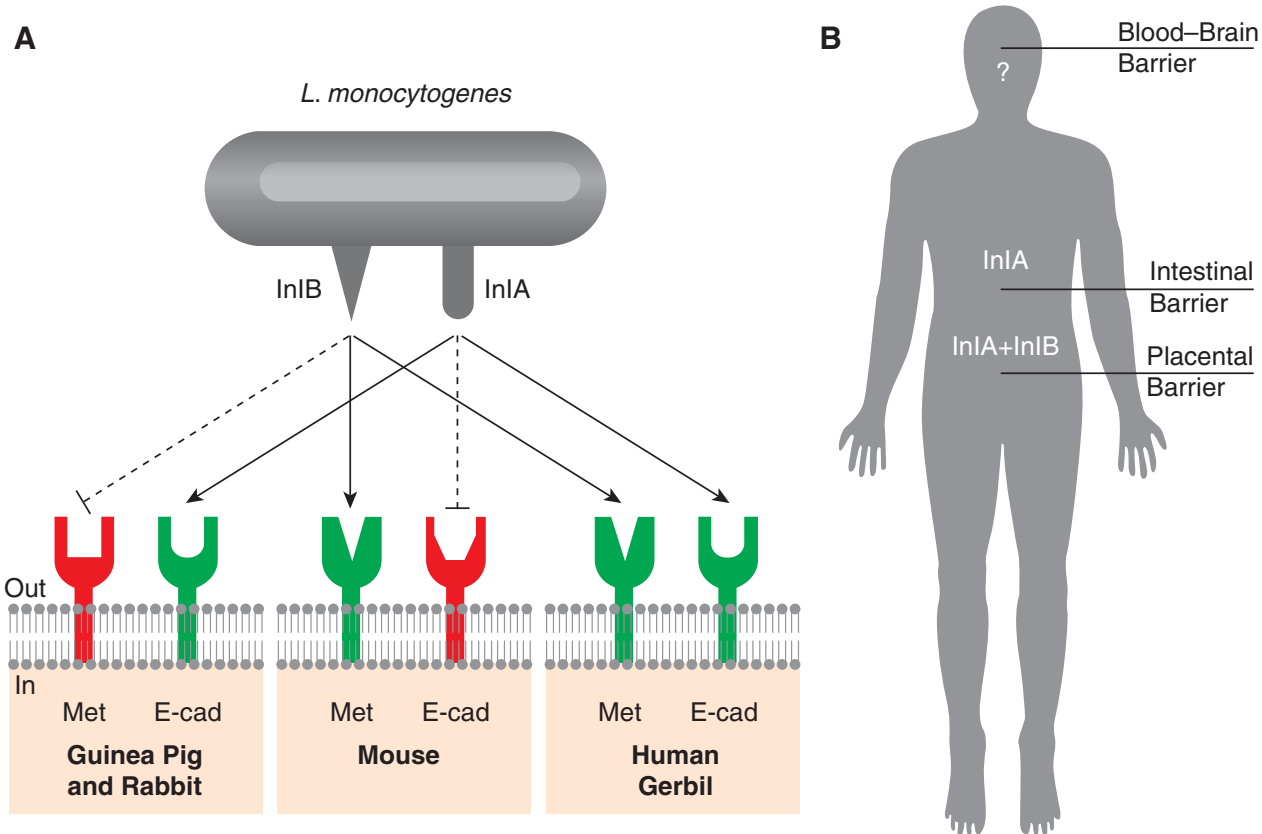

Figure 5. Species specificity of the InlA-E-cadherin interaction $(A)$ The species specificity of the InlA/Ecadherin interaction depends on the presence of a proline in position 16 of the amino-terminal E-cadherin repeat. Species that substitute a glutamate to proline in position 16 of E-cadherin are not sensitive to Listeria infection, as InlA/E-cadherin interactions cannot occur. Based on the observation that some species are not sensitive to InlB, recent studies revealed that species specificity occurs in the case of InlB. (B) InlA/ E-cadherin interaction is fundamental to cross the intestinal barrier, whereas InlB does not play any role at this stage. A coordinated action of the two internalins is necessary to cross the placental barrier, whereas the role of InlA and InlB at the level of the blood-brain barrier is still an unresolved issue. 
fetoplacental listeriosis (Jacquet et al. 2004), and ex vivo experiments using human placenta also showed a role for InlA in placental invasion (Lecuit et al. 2004), this had not been confirmed in vivo until recently. On the contrary, results obtained in pregnant guinea pigs were in apparent contradiction with these human epidemiological and ex vivo findings (Bakardjiev et al. 2005). The important discovery of the species specificity of InlB, for which guinea pig Met does not act as a receptor (Khelef et al. 2006), led to the hypothesis that for InlA to exert its effect at the placental level, InlB was also required. In a search for a small animal model permissive to both InlA and InlB, a natural host for L. monocytogenes, the gerbil, which is naturally susceptible to L. monocytogenes, was tested. Results showed that gerbil primary epithelial cells are permissive to both the InlA and InlB pathways, as human epithelial cells (Disson et al. 2008). Gerbils were then infected orally to assess the role of InlA in the crossing of the intestinal barrier, and as in guinea pigs and transgenic mice expressing human E-cadherin, InlA was shown to contribute to the crossing of the intestinal barrier, but not InlB (Disson et al. 2008). Importantly, wt L. monocytogenes were able to colonize the placenta of pregnant gerbils infected both orally and intravenously, whereas the Listeria mutants that lack either one of the internalins or both $(\Delta i n l A, \Delta i n l B$, or $\Delta i n l A B)$ were far less efficient in crossing the placental barrier and infecting the fetus. Similar data were also obtained in a novel knock-in mouse model where a humanized E16P mouse E-cadherin is expressed in place of mouse E-cadherin (Disson et al. 2008). Together, these data suggest that for the crossing of the placental barrier, both internalin InlA and InlB have to be able to recognize their respective receptors, E-cadherin and Met, to cooperate and allow efficient placental invasion and fetal infection.

\section{E-CADHERIN IS ALSO A RECEPTOR FOR THE OPPORTUNISTIC FUNGUS C. ALBICANS}

E-cadherin has been recently identified as the target for another pathogen surface protein, the C. albicans invasin Als3 (Phan et al. 2007). It has been previously shown that $\mathrm{N}$-cadherin mediates $C$. albicans endocytosis into endothelial cells, a step that is crucial for the development of systemic infections, but the fungal proteins responsible for cadherin engagement were unknown (Phan et al. 2005). The C. albicans als gene family encodes a set of proteins that have a potential role as adhesins and at their amino-termini share a remarkable similarity with Yersinia pseudotuberculosis invasin (Phan et al. 2007). In fact, it has been observed that the protein Als3 is capable of binding E-cadherin and $\mathrm{N}$-cadherin on the surface of epithelial and endothelial cells, respectively, and $a l s 3 \Delta /$ als $3 \Delta$ mutants are no longer internalized within host cells and lose the ability of inducing cellular damage (Phan et al. 2007). Similarly to InlA for Listeria, latex beads coated with recombinant Als3 are efficiently internalized within E-cadherin- and Ncadherin-expressing cells. Interestingly, in silico models of the secondary structure of the Als3 amino terminus revealed a striking similarity with the more distal extracellular domain of E-cadherin, suggesting that, differently from InlA, Als3 may engage E-cadherin at the same sites than those required for adherens junctions formation (Phan et al. 2007).

\section{CONCLUSIONS}

Pathogen mimicry of host natural ligands and subversion of host cellular functions has been a recurrent theme in the relatively new field of cellular microbiology. It has helped decipher complex signaling pathways and unravel novel cellular functions. Studies on the internalization of bacteria such as Listeria, Staphylococcus, and Yersinia, allowed the identification of unsuspected functions for important cell adhesion molecules (Isberg and Barnes 2001; Agerer et al. 2005; Hamon et al. 2006) and opened new perspectives for well-established cellular models (Veiga and Cossart 2005b; Veiga et al. 2007).

Among bacterial pathogens, L. monocytogenes has emerged as a model microorganism for the characterization of key molecular 
mechanisms, both in bacteria and eukaryotic cells: For example, the study of posttranscriptional regulation in Listeria highlighted a novel mechanism of thermoregulation for the promoter PrfA (Johansson et al. 2002); the intracellular actin-based motility of L. monocytogenes has helped unravel the mechanism underlying actin dynamics and cell motility (Welch et al. 1998; Gouin et al. 2005). InlA was the first L. monocytogenes surface protein identified as a mediator of internalization (Gaillard et al. 1991; Mengaud et al. 1996) and the identification of E-cadherin as the cellular receptor for InlA opened the field of adherens junction biology to microbiologists. Since then, L. monocytogenes has been a powerful tool in understanding the complex dynamics of adherens junctions and in building a comprehensive model to describe its functioning.

The development of a humanized smallanimal model, a transgenic mouse expressing human E-cadherin, was critical in establishing the importance of a single amino acid in the EC1 domain of E-cadherin in the stringent species-specificity to L. monocytogenes. It also helped highlight the unique role of InlA in the crossing of the intestinal barrier (Lecuit et al. 2001). Further advances in the development of new animal models led to the important discovery of an interdependent role for the two major internalins, InlA and InlB, in the crossing of the fetoplacental barrier, a key step of the infection (Disson et al. 2008). A current challenge is to understand the molecular mechanisms of the conjugated action of InlA and InlB at the single cell level. Furthermore, recent insights in the cell biology of InlA-mediated infections have also revealed promising interactions between the adhesion and the endocytic machineries (Bonazzi et al. 2008) that deserve further investigation.

\section{REFERENCES}

Agerer F, Lux S, Michel A, Rohde M, Ohlsen K, Hauck CR. 2005. Cellular invasion by Staphylococcus aureus reveals a functional link between focal adhesion kinase and cortactin in integrin-mediated internalisation. J Cell Sci 118: 2189-2200.
Bakardjiev AI, Stacy BA, Portnoy DA. 2005. Growth of Listeria monocytogenes in the guinea pig placenta and role of cell-to-cell spread in fetal infection. J Infect Dis 191: 1889-1897.

Bierne H, Sabet C, Personnic N, Cossart P. 2007. Internalins: A complex family of leucine-rich repeat-containing proteins in Listeria monocytogenes. Microbes Infect 9: 1156-1166.

Bonazzi M, Cossart P. 2006. Bacterial entry into cells: A role for the endocytic machinery. FEBS Lett 580: 2962-2967.

Bonazzi M, Lecuit M, Cossart P. 2009. Listeria monocytogenes internalin and E-cadherin: From structure to pathogenesis. Cell Microbiol 11: 693-702.

Bonazzi M, Veiga E, Pizarro-Cerda J, Cossart P. 2008. Successive post-translational modifications of E-cadherin are required for InlA-mediated internalization of Listeria monocytogenes. Cell Microbiol 10: 2208-2222.

Boyle EC, Finlay BB. 2003. Bacterial pathogenesis: Exploiting cellular adherence. Curr Opin Cell Biol 15: 633-639.

Brembeck FH, Schwarz-Romond T, Bakkers J, Wilhelm S, Hammerschmidt M, Birchmeier W. 2004. Essential role of BCL9-2 in the switch between $\beta$-catenin's adhesive and transcriptional functions. Genes Dev 18: 2225-2230.

Campbell DH, Sutherland RL, Daly RJ. 1999. Signaling pathways and structural domains required for phosphorylation of EMS1/cortactin. Cancer Res 59: 5376-5385.

Cavey M, Rauzi M, Lenne PF, Lecuit T. 2008. A two-tiered mechanism for stabilization and immobilization of E-cadherin. Nature 453: 751-756.

Cossart P, Sansonetti PJ. 2004. Bacterial invasion: The paradigms of enteroinvasive pathogens. Science 304: 242-248.

Delva E, Tucker DK, Kowalczyk AP. 2009. The desmosome. Cold Spring Harb Perspect Biol 1: a002543.

Disson O, Grayo S, Huillet E, Nikitas G, Langa-Vives F, Dussurget O, Ragon M, Le Monnier A, Babinet C, Cossart P, Lecuit M. 2008. Conjugated action of two speciesspecific invasion proteins for fetoplacental listeriosis. Nature 455: 1114-1118.

Drees F, Pokutta S, Yamada S, Nelson WJ, Weis WI. 2005. $\alpha$-catenin is a molecular switch that binds E-cadherin$\beta$-catenin and regulates actin-filament assembly. Cell 123: 903-915.

Dubois T, Paleotti O, Mironov AA, Fraisier V, Stradal TE, De Matteis MA, Franco M, Chavrier P. 2005. Golgi-localized GAP for Cdc42 functions downstream of ARF1 to control Arp2/3 complex and F-actin dynamics. Nat Cell Biol 7: 353-364.

Dupre-Crochet S, Figueroa A, Hogan C, Ferber EC, Bialucha CU, Adams J, Richardson EC, Fujita Y. 2007. Casein kinase 1 is a novel negative regulator of E-cadherin-based cell-cell contacts. Mol Cell Biol 27: 3804-3816.

Fujita Y, Krause G, Scheffner M, Zechner D, Leddy HE, Behrens J, Sommer T, Birchmeier W. 2002. Hakai, a c-Cbl-like protein, ubiquitinates and induces endocytosis of the E-cadherin complex. Nat Cell Biol 4: 222-231. 
Gaillard JL, Berche P, Frehel C, Gouin E, Cossart P. 1991 Entry of L. monocytogenes into cells is mediated by internalin, a repeat protein reminiscent of surface antigens from gram-positive cocci. Cell 65: 1127-1141.

Gaillard JL, Berche P, Mounier J, Richard S, Sansonetti P. 1987. In vitro model of penetration and intracellular growth of Listeria monocytogenes in the human enterocyte-like cell line Caco-2. Infect Immun 55: 2822-2829.

Gottardi CJ, Gumbiner BM. 2004. Distinct molecular forms of $\beta$-catenin are targeted to adhesive or transcriptional complexes. J Cell Biol 167: 339-349.

Gouin E, Welch MD, Cossart P. 2005. Actin-based motility of intracellular pathogens. Curr Opin Microbiol 8: 35-45.

Green KJ, Gaudry CA. 2000. Are desmosomes more than tethers for intermediate filaments? Nat Rev Mol Cell Biol 1: 208-216.

Hamon M, Bierne H, Cossart P. 2006. Listeria monocytogenes: A multifaceted model. Nat Rev Microbiol 4: 423-434.

Hauck CR, Agerer F, Muenzner P, Schmitter T. 2006. Cellular adhesion molecules as targets for bacterial infection. Eur Cell Biol 85: 235-242.

Heuberger J, Birchmeier W. 2009. Interplay of cadherinmediated cell adhesion and canonical Wnt signaling. Cold Spring Harb Perspect Biol 2: a002915.

Huang C, Liu J, Haudenschild CC, Zhan X. 1998. The role of tyrosine phosphorylation of cortactin in the locomotion of endothelial cells. J Biol Chem 273: 25770-25776.

Huang C, Ni Y, Wang T, Gao Y, Haudenschild CC, Zhan X. 1997. Down-regulation of the filamentous actin crosslinking activity of cortactin by Src-mediated tyrosine phosphorylation. J Biol Chem 272: 13911-13915.

Isberg RR, Barnes P. 2001. Subversion of integrins by enteropathogenic Yersinia. J Cell Sci 114: 21-28.

Jacquet C, Doumith M, Gordon JI, Martin PM, Cossart P, Lecuit M. 2004. A molecular marker for evaluating the pathogenic potential of foodborne Listeria monocytogenes. J Infect Dis 189: 2094-2100.

Johansson J, Mandin P, Renzoni A, Chiaruttini C, Springer M, Cossart P. 2002. An RNA thermosensor controls expression of virulence genes in Listeria monocytogenes. Cell 110: 551-561.

Jonquieres R, Bierne H, Mengaud J, Cossart P. 1998. The inlA gene of Listeria monocytogenes LO28 harbors a nonsense mutation resulting in release of internalin. Infect Immun 66: 3420-3422.

Joshi B, Strugnell SS, Goetz JG, Kojic LD, Cox ME, Griffith OL, Chan SK, Jones SJ, Leung SP, Masoudi H, et al. 2008. Phosphorylated caveolin-1 regulates Rho/ROCKdependent focal adhesion dynamics and tumor cell migration and invasion. Cancer Res 68: 8210-8220.

Karecla PI, Green SJ, Bowden SJ, Coadwell J, Kilshaw PJ. 1996. Identification of a binding site for integrin $\alpha \mathrm{E} \beta 7$ in the N-terminal domain of E-cadherin. J Biol Chem 271: 30909-30915.

Khelef N, Lecuit M, Bierne H, Cossart P. 2006. Species specificity of the Listeria monocytogenes InlB protein. Cell Microbiol 8: 457-470.

Kobe B, Deisenhofer J. 1994. The leucine-rich repeat: Aversatile binding motif. Trends Biochem Sci 19: 415-421.
Kohmura N, Senzaki K, Hamada S, Kai N, Yasuda R, Watanabe M, Ishii $\mathrm{H}$, Yasuda M, Mishina M, Yagi T 1998. Diversity revealed by a novel family of cadherins expressed in neurons at a synaptic complex. Neuron 20: 1137-1151.

Lecuit M. 2007. Human listeriosis and animal models. Microbes Infect 9: 1216-1225.

Lecuit M, Dramsi S, Gottardi C, Fedor-Chaiken M, Gumbiner B, Cossart P. 1999. A single amino acid in E-cadherin responsible for host specificity towards the human pathogen Listeria monocytogenes. Embo J 18: 3956-3963.

Lecuit M, Hurme R, Pizarro-Cerda J, Ohayon H, Geiger B, Cossart P. 2000. A role for $\alpha$-and $\beta$-catenins in bacterial uptake. Proc Natl Acad Sci 97: 10008-10013.

Lecuit M, Nelson DM, Smith SD, Khun H, Huerre M, Vacher-Lavenu MC, Gordon JI, Cossart P. 2004. Targeting and crossing of the human maternofetal barrier by Listeria monocytogenes: Role of internalin interaction with trophoblast E-cadherin. Proc Natl Acad Sci 101: 6152-6157.

Lecuit M, Ohayon H, Braun L, Mengaud J, Cossart P. 1997. Internalin of Listeria monocytogenes with an intact leucine-rich repeat region is sufficient to promote internalization. Infect Immun 65: 5309-5319.

Lecuit M, Vandormael-Pournin S, Lefort J, Huerre M, Gounon P, Dupuy C, Babinet C, Cossart P. 2001. A transgenic model for listeriosis: Role of internalin in crossing the intestinal barrier. Science 292: 1722-1725.

Martinez-Quiles N, Ho HY, Kirschner MW, Ramesh N, Geha RS. 2004. Erk/Src phosphorylation of cortactin acts as a switch on-switch off mechanism that controls its ability to activate N-WASP. Mol Cell Biol 24: 5269-5280.

McCrea PD, Gu D, Balda M. 2009. Junctional music that the nucleus hears. Cold Spring Harb Perspect Biol 1: a002923.

Meng W, Takeichi M. 2009. Adherens junction: Molecular architecture and regulation. Cold Spring Harb Perspect Biol 1: a002899.

Mengaud J, Ohayon H, Gounon P. 1996. E-cadherin is the receptor for internalin, a surface protein required for entry of L. monocytogenes into epithelial cells. Cell 84: 923-932.

Ozawa M, Ringwald M, Kemler R. 1990. Uvomorulincatenin complex formation is regulated by a specific domain in the cytoplasmic region of the cell adhesion molecule. Proc Natl Acad Sci 87: 4246-4250.

Patel SD, Chen CP, Bahna F, Honig B, Shapiro L. 2003. Cadherin-mediated cell-cell adhesion: Sticking together as a family. Curr Opin Struct Biol 13: 690-698.

Phan QT, Fratti RA, Prasadarao NV, Edwards JE Jr, Filler SG. 2005. N-cadherin mediates endocytosis of Candida albicans by endothelial cells. J Biol Chem 280: 10455-10461.

Phan QT, Myers CL, Fu Y, Sheppard DC, Yeaman MR, Welch WH, Ibrahim AS, Edwards JE, Filler SG. 2007. Als3 is a Candida albicans invasin that binds to cadherins and induces endocytosis by host cells. PLoS Biol 5: e64. doi: 10.1371/journal.pbio.0050064.

Pizarro-Cerda J, Cossart P. 2006. Bacterial adhesion and entry into host cells. Cell 124: 715-727. 
Pizarro-Cerda J, Jonquieres R, Gouin E, Vandekerckhove J, Garin J, Cossart P. 2002. Distinct protein patterns associated with Listeria monocytogenes InlA- or InlBphagosomes. Cell Microbiol 4: 101-115.

Ranscht B, Dours-Zimmermann MT. 1991. T-cadherin, a novel cadherin cell adhesion molecule in the nervous system lacks the conserved cytoplasmic region. Neuron 7: 391-402.

Reynolds AB. 2007. p120-catenin: Past and present. Biochim Biophys Acta 1773: 2-7.

Rhee J, Buchan T, Zukerberg L, Lilien J, Balsamo J. 2007. Cables links Robo-bound Abl kinase to $\mathrm{N}$-cadherin bound $\beta$-catenin to mediate Slit-induced modulation of adhesion and transcription. Nat Cell Biol 9: 883-892.

Roura S, Miravet S, Piedra J, Garcia de Herreros A, Dunach M. 1999. Regulation of E-cadherin/Catenin association by tyrosine phosphorylation. J Biol Chem 274: 36734-36740.

Schubert WD, Urbanke C, Ziehm T, Beier V, Machner MP, Domann E, Wehland J, Chakraborty T, Heinz DW. 2002. Structure of internalin, a major invasion protein of Listeria monocytogenes, in complex with its human receptor E-cadherin. Cell 111: 825-836.

Shapiro L, Weis WI. 2009. Structure and biochemistry of cadherins and catenins. Cold Spring Harb Perspect Biol 1: a003053.

Sousa S, Cabanes D, Archambaud C, Colland F, Lemichez E, Popoff M, Boisson-Dupuis S, Gouin E, Lecuit M, Legrain P, et al. 2005. ARHGAP10 is necessary for $\alpha$-catenin recruitment at adherens junctions and for Listeria invasion. Nat Cell Biol 7: 954-960.

Sousa S, Cabanes D, Bougneres L, Lecuit M, Sansonetti P, Tran-Van-Nhieu G, Cossart P. 2007. Src, cortactin and Arp2/3 complex are required for E-cadherin-mediated internalization of Listeria into cells. Cell Microbiol 9: 2629-2643.

Sousa S, Cabanes D, El-Amraoui A, Petit C, Lecuit M, Cossart P. 2004. Unconventional myosin VIIa and vezatin, two proteins crucial for Listeria entry into epithelial cells. J Cell Sci 117: 2121-2130.

Troyanovsky S. 2005. Cadherin dimers in cell-cell adhesion. Eur J Cell Biol 84: 225-233.

Vasioukhin V, Fuchs E. 2001. Actin dynamics and cell-cell adhesion in epithelia. Curr Opin Cell Biol 13: 76-84.
Veiga E, Cossart P. 2005a. Ubiquitination of intracellular bacteria: A new bacteria sensing system? Trends Cell Biol 15: $2-5$.

Veiga E, Cossart P. 2005b. Listeria hijacks the clathrindependent endocytic machinery to invade mammalian cells. Nat Cell Biol 7: 894-900.

Veiga E, Guttman JA, Bonazzi M, Boucrot E, Toledo-Arana A, Lin AE, Enninga J, Pizarro-Cerda J, Finlay BB, Kirchhausen T, et al. 2007. Invasive and adherent bacterial pathogens co-opt host clathrin for infection. Cell Host Microbe 2: 340-351.

Vestweber D, Winderlich M, Cagna G, Nottebaum AF. 2009. Cell adhesion dynamics at endothelial junctions: VE-cadherin as a major player. Trends Cell Biol 19: 8-15.

Weed SA, Du Y, Parsons JT. 1998. Translocation of cortactin to the cell periphery is mediated by the small GTPase Rac1. J Cell Sci 111: 2433-2443.

Welch MD, Rosenblatt J, Skoble J, Portnoy DA, Mitchison TJ. 1998. Interaction of human Arp2/3 complex and the Listeria monocytogenes ActA protein in actin filament nucleation. Science 281: 105-108.

Wilde A, Beattie EC, Lem L, Riethof DA, Liu SH, Mobley WC, Soriano P, Brodsky FM. 1999. EGF receptor signaling stimulates SRC kinase phosphorylation of clathrin, influencing clathrin redistribution and EGF uptake. Cell 96: $677-687$.

Wollert T, Heinz DW, Schubert WD. 2007a. Thermodynamically reengineering the listerial invasion complex InlA/E-cadherin. Proc Natl Acad Sci 104: 13960-13965.

Wollert T, Pasche B, Rochon M, Deppenmeier S, van den Heuvel J, Gruber AD, Heinz DW, Lengeling A, Schubert WD. 2007b. Extending the host range of Listeria monocytogenes by rational protein design. Cell 129: 891-902.

Wu S, Lim KC, Huang J, Saidi RF, Sears CL. 1998. Bacteroides fragilis enterotoxin cleaves the zonula adherens protein, E-cadherin. Proc Natl Acad Sci 95: 14979-14984.

Yamada S, Pokutta S, Drees F, Weis WI, Nelson WJ. 2005. Deconstructing the cadherin-catenin-actin complex. Cell 123: 889-901.

Zeng G, Apte U, Micsenyi A, Bell A, Monga SP. 2006. Tyrosine residues 654 and 670 in $\beta$-catenin are crucial in regulation of Met- $\beta$-catenin interactions. Exp Cell Res 312: 3620-3630. 


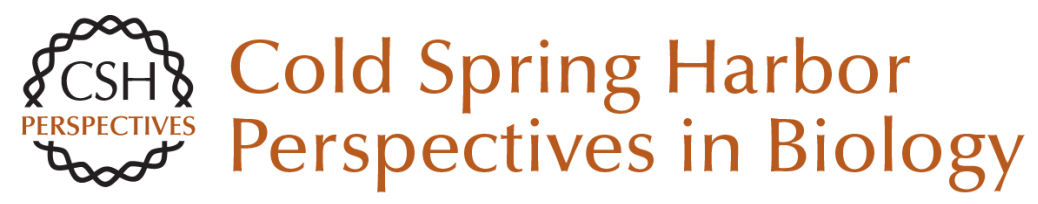

\title{
Listeria monocytogenes Internalin and E-cadherin: From Bench to Bedside
}

\author{
Matteo Bonazzi, Marc Lecuit and Pascale Cossart
}

Cold Spring Harb Perspect Biol 2009; doi: 10.1101/cshperspect.a003087 originally published online September 16, 2009

\section{Subject Collection Cell-Cell Junctions}

Vascular Endothelial (VE)-Cadherin, Endothelial

Adherens Junctions, and Vascular Disease Maria Grazia Lampugnani, Elisabetta Dejana and Costanza Giampietro

Adherens Junctions and Desmosomes Coordinate Mechanics and Signaling to Orchestrate Tissue Morphogenesis and Function: An Evolutionary Perspective Matthias Rübsam, Joshua A. Broussard, Sara A. Wickström, et al.

Cell-Cell Contact and Receptor Tyrosine Kinase Signaling Christine Chiasson-MacKenzie and Andrea I. McClatchey

Hold Me, but Not Too Tight--Endothelial Cell-Cell Junctions in Angiogenesis

Anna Szymborska and Holger Gerhardt

Connexins and Disease

Mario Delmar, Dale W. Laird, Christian C. Naus, et al.

Cell Junctions in Hippo Signaling Ruchan Karaman and Georg Halder
Signaling by Small GTPases at Cell-Cell Junctions: Protein Interactions Building Control and Networks Vania Braga

Making Connections: Guidance Cues and Receptors at Nonneural Cell-Cell Junctions lan V. Beamish, Lindsay Hinck and Timothy E. Kennedy

The Cadherin Superfamily in Neural Circuit Assembly James $D$. Jontes

Mechanosensing and Mechanotransduction at Cell-Cell Junctions Alpha S. Yap, Kinga Duszyc and Virgile Viasnoff

Beyond Cell-Cell Adhesion: Sensational

Cadherins for Hearing and Balance Avinash Jaiganesh, Yoshie Narui, Raul Araya-Secchi, et al.

Cell-Cell Junctions Organize Structural and Signaling Networks Miguel A. Garcia, W. James Nelson and Natalie Chavez

For additional articles in this collection, see http://cshperspectives.cshlp.org/cgi/collection/

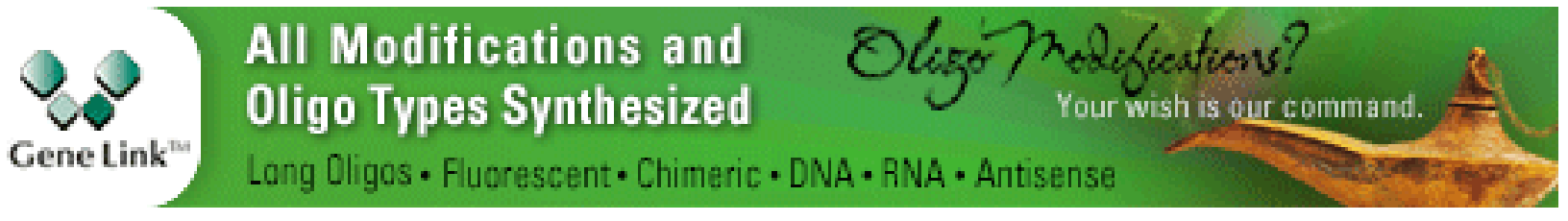


Loss of E-Cadherin-Dependent Cell-Cell Adhesion and the Development and Progression of Cancer Heather C. Bruner and Patrick W.B. Derksen

Desmosomes and Intermediate Filaments: Their Consequences for Tissue Mechanics

Mechthild Hatzfeld, René Keil and Thomas M. Magin
Cell Biology of Tight Junction Barrier Regulation and Mucosal Disease

Aaron Buckley and Jerrold R. Turner

Integration of Cadherin Adhesion and

Cytoskeleton at Adherens Junctions

René Marc Mège and Noboru Ishiyama

For additional articles in this collection, see http://cshperspectives.cshlp.org/cgi/collection/

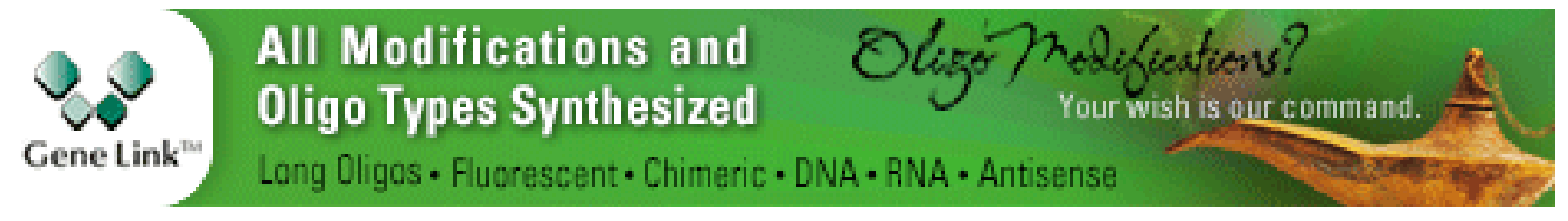

Copyright @ 2009 Cold Spring Harbor Laboratory Press; all rights reserved 